\title{
Issues in Wearable Mobile Sensor Platform for Lower Limb Prosthetic Users
}

\author{
Neha Mathur*a, Ivan Glesk ${ }^{\mathrm{a}}$, Arjan Buis ${ }^{\mathrm{b}}$ \\ ${ }^{a}$ Department of Electronic and Electrical Engineering, University of Strathclyde, Glasgow, United Kingdom \\ ${ }^{b}$ Department of Biomedical Engineering, University of Strathclyde, Glasgow, United Kingdom \\ e-mail:neha.mathur@strath.ac.uk
}

\begin{abstract}
Around the world health services are looking for ways of improving patient care for amputees suffering from diabetes, while at the same time reducing costs. These include remote monitoring of physiological data such as temperature, gait, heart rate, blood pressure, blood oxygenation $(\mathrm{SpO} 2)$, respiration and glucose levels. The ehealth wearable communication systems show promise in delivering improvements in patient care while at the same time reducing both the demand for resources and the financial burden on healthcare systems. These systems have the capability of monitoring, logging and transmitting the patient data to a central health authority. Depending on the patient, it is often critical that the monitoring system reliability is high to deliver the appropriate patient care and ensure patient safety. These wearable systems that would be placed in the prosthesis of the amputee are dependent on the battery power to drive them for continuous monitoring followed by data transmission. However, if improperly designed will rapidly deplete the battery energy making the system short lived and quickly prone to failure. Also, weight and size of the battery is a concern, as a heavier battery could induce discomfort to the amputee. In this paper, transmission system design and methods to reduce power consumption will be discussed using the example of prosthetic socket compatible temperature and gait monitoring data systems.
\end{abstract}

Keywords: Battery power, Data monitoring, Data transmission, Healthcare, Prosthesis, Wearable technologies.

\section{INTRODUCTION}

Recent advances in internet and mobile communications devices, as well as ubiquitous computing have fostered a tremendous growth in the field of wearable technologies. They have opened countless possibilities of monitoring patients in the field over extended periods of time. This is particularly relevant when clinicians/physicians want to monitor individuals undergoing rehabilitation for whom interventions need to be assessed in the home and outdoor environment. It would enable them to support early detection of abnormal conditions and prevention of serious consequences. Prosthetic limb users especially those suffering from Type 2 Diabetes Mellitus (T2DM) can greatly benefit from continuous monitoring as the common complications of T2DM are infection, gangrene, Charcot foot disease and amputation. It is estimated that each year in the United States, there are more than 80,000 lower-limb amputations because of diabetics. Hence, many patients can benefit from continuous monitoring of their residual limb tissue health as a part of diagnostic procedure, routine maintenance or during supervised recovery from a surgical procedure.

Wearable systems for prosthetic users have to be designed such that the sensors are minimally obtrusive and sensitive enough to reliably record movement or physiological signals like temperature, gait, heart rate, blood pressure and blood oxygenation ( $\mathrm{SpO} 2)$ levels . This is especially significant as problems could exist having the sensor wires coming out of the socket and through possible skin irritation caused by the undesirable contact of the measurement device on the residual limb skin. Another challenge is the development of a system which unobtrusively gathers data from multiple wearable sensors and transfers this information periodically to a central health authority database server via a wireless transfer protocol. The data sets recorded using these systems are then processed using machine learning algorithms. These algorithms must be trainable without requiring extensive human supervision to extract clinically relevant information from the data. The knowledge would be useful to detect biomarkers of possible worsening of the patient's clinical situation or they are assessed to see the impact of the clinical interventions [1], [2].

However, since wearable systems are solely dependent on battery power, continuous monitoring followed by data transmission if improperly designed will rapidly deplete the battery energy making the system short lived and quickly prone to failure. Ideally the battery should be able to provide enough charge to power the device for extended periods. However, the current battery technology is unable to deliver the improvements in capacity currently demanded by the development of smaller, more resource hungry devices. In summary while devices are reducing in size, the battery size cannot be reduced without incurring a severe penalty in the level of charge it can hold. Batteries could be quite easily the biggest bottleneck in the advancement of wearable technologies [3].

Our approach was to develop a mobile sensor platform for lower limb prosthetic users using an Arduino board which could encompass sensors for temperature, gait and orientation measurements. The platform is investigated for its power consumption when transmitting the above mentioned sensors data to an open cloud infrastructure through three different modes of communication - Bluetooth, Wi-Fi and GSM protocol at a fixed sampling rate. 
The platform is supplied with different batteries in each scenario and the results are compared for the same. The aim of this study is to examine the battery technology that would be most suitable and long lasting for our wearable technology design.

\section{WEARABLE SENSOR PLATFORM DESIGN}

The mobile sensor platform developed is capable of measuring temperature, acceleration and orientation of the amputee subject at a user defined sampling rate. There is two way communication between the mobile sensor platform and the remote monitoring site; firstly, the measured data being transmitted to a cloud server periodically where it can be accessed by healthcare personnel using internet and secondly, the status of the sensor platform being remotely monitored at the healthcare system end where commands for control can be relayed from the internet to input/output board by the cloud. The data can simultaneously also be received by an amputee user on the mobile via an app and stored on the mobile's SD card. The Arduino platform offers versatility to the user in data transmission. Any of the three transmission protocols can be used - Bluetooth, WiFi or GSM; but it should be noted that the power requirement of each of them is quite different. There is also a provision to locally store the data onto a SD card on the Arduino board where it can be accessed locally by a technician. Fig. 1 shows the schematic of the mobile wearable sensor design.

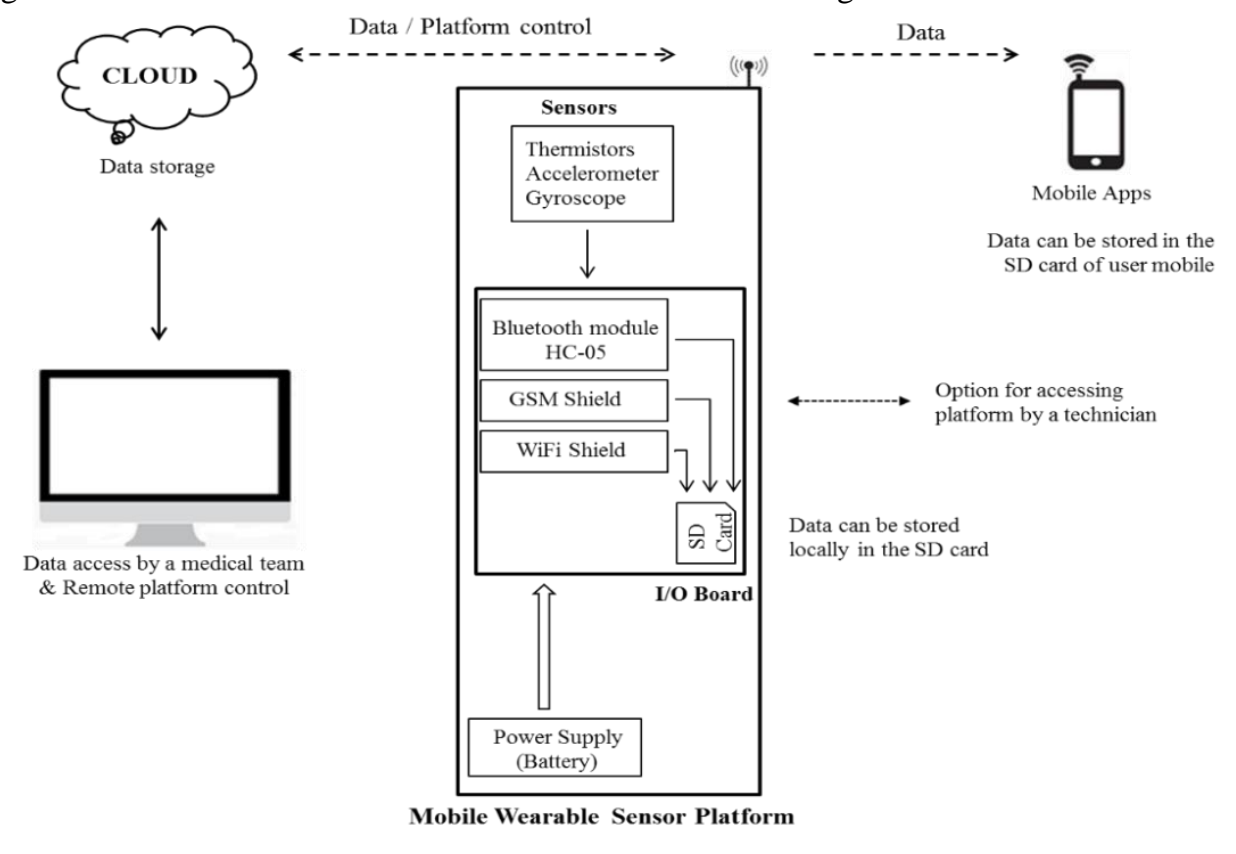

\section{Figure 1. Architecture of the mobile wearable sensor platform with in-built three modes of communication to a central health database accessed via web or mobile apps.}

The core of the wearable sensor platform is an Arduino Uno board, which runs on 3.3-5V and comprises an ATmega328 microcontroller, a reset switch, indicator LEDs, 32 Kbytes of program memory, an 16-MHz processor, a 10-bit analog-to-digital converter, and $14 \mathrm{I} / \mathrm{O}$ pins, including 6 analog inputs and 6 pulse-width modulation (PWM) outputs. The board needs to be supplied with external battery power in the range of 7-12V, which is then stepped down using a voltage regulator in built in the board. For the initial deployment, a negative temperature coefficient (NTC) thermistor along with MPU 6050 which is a triple axis accelerometer and triple axis gyroscope are used as the sensor inputs to the board.

The Arduino board can be interfaced with a Bluetooth module, a GSM shield or a Wi-Fi shield. The Bluetooth module HC-05 is an easy to use SPP (Serial Port Protocol) module, designed for transparent wireless serial connection setup. Serial port Bluetooth module is fully qualified Bluetooth V2.0+EDR (Enhanced Data Rate) $3 \mathrm{Mbps}$ modulation with complete $2.4 \mathrm{GHz}$ radio transceiver and baseband. It uses CSR Bluecore 04-External single chip Bluetooth system with CMOS technology and with AFH (Adaptive Frequency Hopping Feature). It has the footprint as small as $12.7 \mathrm{~mm} \times 27 \mathrm{~mm}$. Its low power operation at $1.8 \mathrm{~V}$ makes it even more compatible for use in this design.

The Wi-Fi shield for the Arduino connects to the internet wirelessly using the 802.11 wireless specifications. It is based on the HDG204 Wireless LAN 802.11b/g System in-Package. An AT32UC3 provides a network (IP) stack capable of both TCP and UDP. The Wi-Fi shield connects to an Arduino board using long wire-wrap headers which extend through the shield. The current draw of the Arduino is roughly 25-50mA whereas that of the Wi-Fi shield will be 60-500mA depending upon how much network traffic is sending/receiving. Thus, the power consumption of this shield is huge and can drain out the battery fairly quickly. 
The Arduino GSM shield connects the Arduino to the internet using the GPRS wireless network. The shield uses a radio modem M10 by Quectel. The M10 is a Quad-band GSM/GPRS modem that works at frequencies GSM850MHz, GSM900MHz, DCS1800MHz and PCS1900MHz. It supports TCP/UDP and HTTP protocols through a GPRS connection. GPRS data downlink and uplink transfer speed maximum is 85.6 kbps. However, the modem can pull up to $2 \mathrm{~A}$ of current at peak usage, which can occur during data transmission and requires $700-1000 \mathrm{~mA}$ of current draw at other times [4]. This is of concern, as in the realisation of the above requirements are the power consumption of the device and the longevity of the battery between charges.

\section{METHODOLOGY}

As seen from the design of the wearable sensor platform in the previous section, it basically consists of a microcontroller based unit for control and pre-processing of the sensors data; and a transmitter and a smart phone for data processing and data transfer via internet to a cloud based server. But for continuous monitoring, which could provide a more realistic view of the amputee's lower limb status which would otherwise go unrecorded, a reliable source of power i.e. the battery needs to be identified.

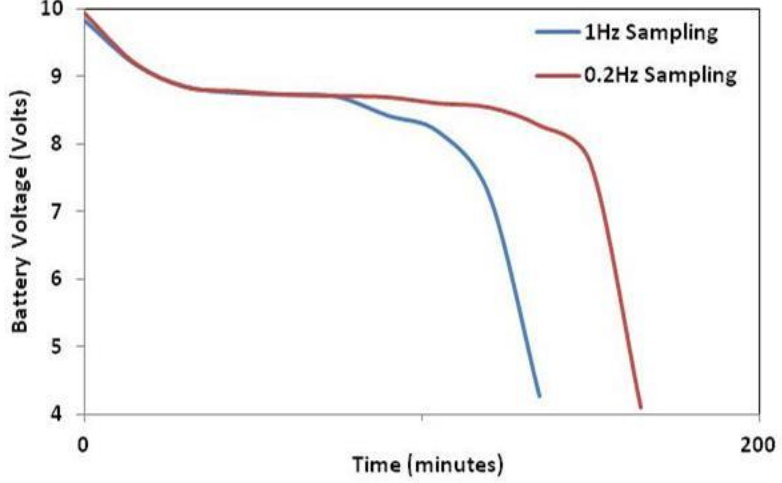

(a)

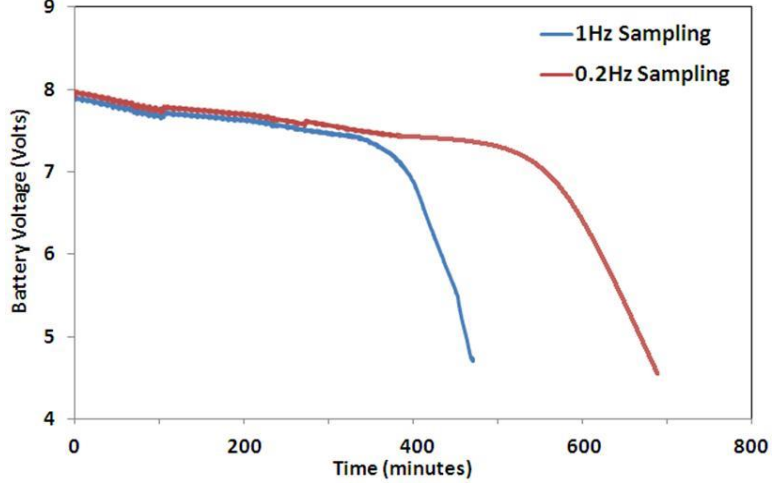

(b)

Figure 2. Rate of depletion of batteries when data transmitted to the cloud is via Bluetooth

(a) NiMH battery (b) Li-ion battery

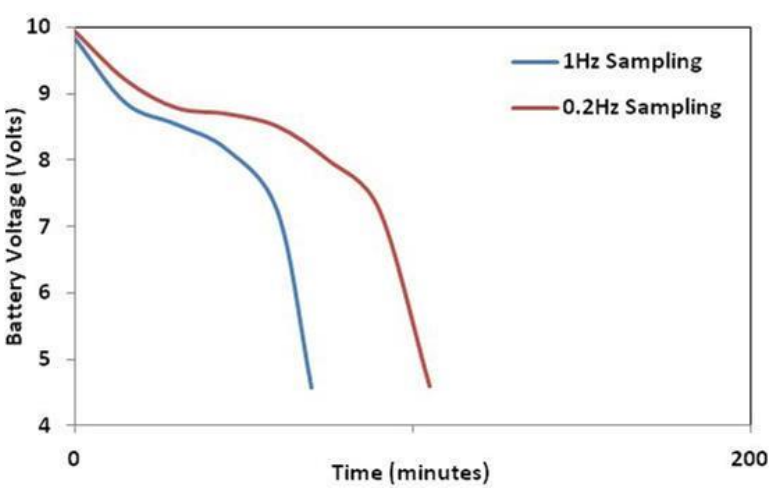

(a)

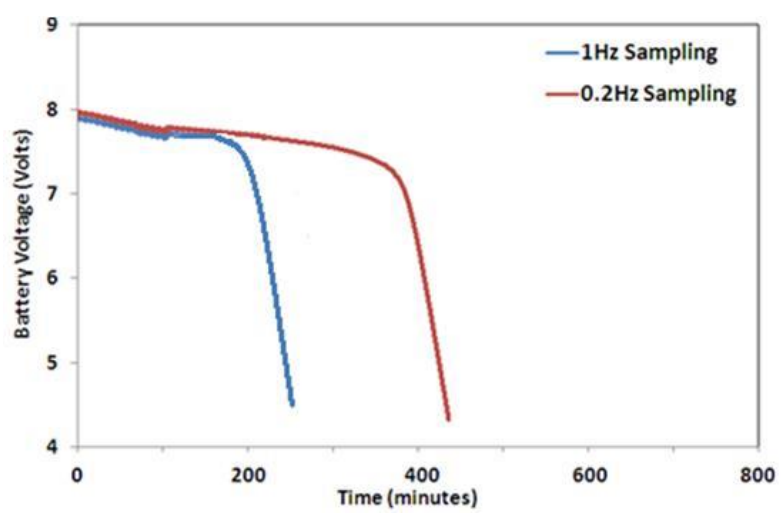

(b)

Figure 3. Rate of depletion of batteries when data transmitted to the cloud is via Wi-Fi shield (a) NiMH battery (b) Li-ion battery

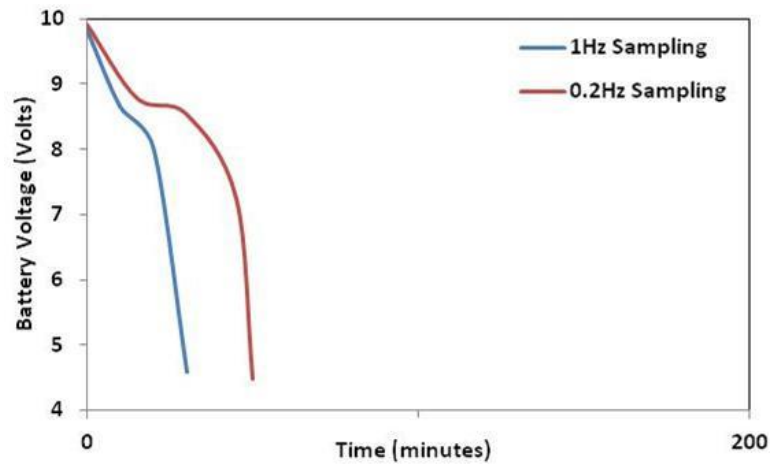

(a)

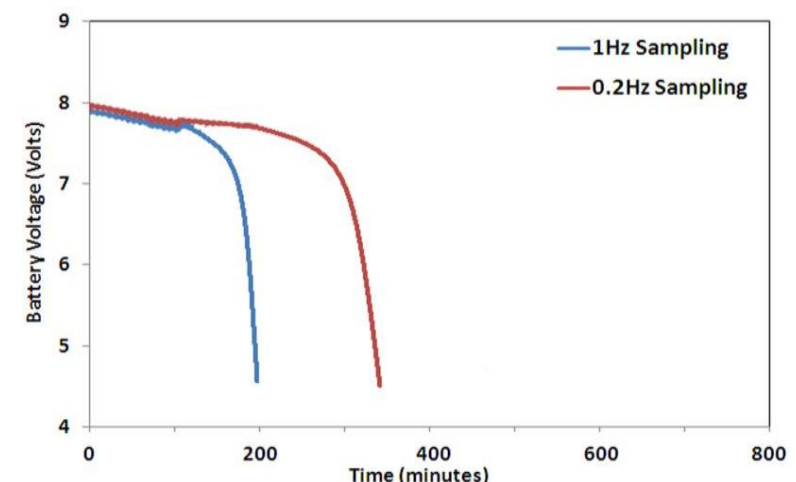

(b)

Figure 4. Rate of depletion of batteries when data transmitted to the cloud is via GSM shield

(a) NiMH battery (b) Li-ion battery 
The mobile sensor platform was first interfaced with the Bluetooth module and temperature and gait data was sent over to the cloud server at a sampling rate of $1 \mathrm{~Hz}$ constantly. The battery depletion rate was continuously monitored and logged using a custom built interface in LabView. This test was run until the battery level was exhausted to the minimum threshold voltage of $5 \mathrm{~V}$ which is the minimum for the Arduino board to operate. The batteries involved in this study were the NiMH rechargeable battery $(9 \mathrm{~V})$ and Li-ion rechargeable battery $(3.7 \mathrm{~V}$ $3000 \mathrm{mAh}$ each; used two in series). This test was repeated for each of the batteries and the rate of depletion was observed. The above protocol was repeated for the other communication methods namely via the Wi-Fi and GSM shields. To study the effect of sampling rate on the battery life, the above routine was repeated for a decreased sampling rate of $0.2 \mathrm{~Hz}$. The results from all the cases were then compared to infer the best possible battery technology which could enable providing consistent data for a long period of time. The data was uploaded on a private user page on the cloud server Xively and the database was accessed on the mobile through a dedicated Xively Viewer mobile app and on the web through the webpage.

From Fig 2, 3 and 4 the depletion rate of both NiMH and Li-ion battery can be observed. It can be seen that the $\mathrm{NiMH}$ battery is not able to support the high current draw demanded by the sensor platform and it can operate for a maximum of three hours when data is transmitted by Bluetooth at $0.2 \mathrm{~Hz}$. Hence, it is not deemed suitable for medical sensor platform applications as with high drain-rate usage (1-4C), the change in shape in the voltage curve with the more rounded "knee" to the curve means that an arbitrary $0.9 \mathrm{~V} /$ battery cut-off may be premature, leaving a significant fraction of the battery capacity untapped. The Li-ion on the other hand is a high density energy battery with the discharge curve being almost flat [5]. This simplifies the design of the application in which the battery is used since the supply voltage stays reasonably constant throughout the discharge cycle. When the sampling rate is decreased from $1 \mathrm{~Hz}$ to $0.2 \mathrm{~Hz}$, the battery life is significantly increased in each mode of communication. However, when the data is transferred via Wi-Fi or GSM shield the battery life, none of the two batteries could last for more than 6 hours, making it the system short lived. Reducing the data sampling rate maybe a solution for reducing the battery consumption but when used to monitor critical amputee data, this may prove to be a detriment in identifying the biomarkers in tissue health.

\section{CONCLUSIONS}

It is observed from the results in Fig 4 that the rate of depletion of all the batteries is greatest when the Arduino board is interfaced with the GSM shield. This is followed by the Wi-Fi shield and the power consumption is least when data is being transmitted via the Bluetooth module. It implies that probably Bluetooth communication is most power effective of all. It is practically impossible to power up the sensor platform continuously for a whole day when sampling at $1 \mathrm{~Hz}$ even with Li-ion battery. Although, balanced Lithium-polymer batteries have higher capacity rating with the weight energy density to be $170 \mathrm{Wh} / \mathrm{kg}$ as compared to $\mathrm{NiMH}$ and Li-ion with $80 \mathrm{Wh} / \mathrm{kg}$ and $125 \mathrm{Wh} / \mathrm{kg}$ respectively; but are unsuitable as the weight of balanced Lithium-polymer batteries is $213 \mathrm{~g}$ making it five times heavier than its counterparts. Hence, in order for the wearable technology platform to be compact, portable and wearable by the amputee in their prosthesis, the battery size is of great importance as a heavier power supply could be a source of discomfort and induce limping/gait issues in the amputees.

In the meantime, significant reductions in power consumption may be achieved by, for example reducing the duty cycle of sensors, reducing the data sampling rate, reducing the MCU processor clock speed; and reducing the amount of data transmitted between MCU and smart-phone and smart-phone and server. However, careful consideration must be made between the relative importance of balancing the conflicting requirements of, on the one hand providing sufficient battery power supply life; and on the other, providing a sufficient level of information to ensure reliable diagnosis and decision making.

\section{ACKNOWLEDGEMENTS}

This work was supported by the Engineering and Physical Sciences Research Council under the Doctoral Training Grant. This investigation was implemented following ethical approval granted by the University of Strathclyde Ethics Committee (Ref UEC13/04).

\section{REFERENCES}

[1] A. Davidson, N. Mathur, I. Glesk, A. Buis: Power Supply Issues in e-Health Monitoring Applications, in Proc. ICREPQ 2015, Cordoba, Spain, March 2015.

[2] V. Chan, P. Ray, and N. Parameswaran: Mobile eHealth monitoring: an agent-based approach, IET Communications, vol. 2, no. 2, pp. 223-230, Feb. 2008.

[3] Xiaoming Zhao et al: A Telemedicine System for Wireless Home Healthcare Based on Bluetooth ${ }^{\mathrm{TM}}$ and the Internet, in Telemedicine Journal and e-Health, vol. 10, no. 2, pp. 110-116, Nov. 2004.

[4] [online].http://arduino.cc/ (Dec. 2014).

[5] [online].http://batteryuniversity.com/learn/article/the_high_power_lithium_ion/ (Nov. 2014). 